\begin{abstract}
Case studies have four functional roles which, if more widely embraced, can help to advance theory and methodology in the study of emerging adults. These functions are: case-based theory development, individual-level prediction testing, theory exemplification, and idiographic psychobiography. We describe these functions and provide examples of how each one can add depth, richness and rigour to the burgeoning theory and research on emerging adulthood. We also discuss specifiability (the capacity of a theory to make predictions about, explain and interpret individuals) as a criterion of validity, to be considered as equivalent in status to the external validity criterion of generalizability. Finally we consider the protocols for case study sampling, including intensity sampling, deviant case sampling and significant case sampling.
\end{abstract}

\title{
Keywords
}

Case study, theory, psychobiography, within-case analysis, idiographic, specifiability, emerging adulthood 


\section{Four functional roles for case studies in emerging adulthood research}

A case study is any research initiative in which the investigator focuses data collection, analysis and reporting on a single person, event, dyad, organization or social group. Case study research can be qualitative and/or quantitative, and correspondingly there are distinct single-case traditions in both paradigms. A case study may be reported on its own, or within a report that presents a series of case studies and compares them.

The case study has been a peripheral methodology in psychology since the Second World War, but has been increasing in popularity recently. Central to this has been the renaissance of single-case experimental designs that investigate intra-individual change and present data caseby-case without averaging into aggregates (Barlow \& Nock, 2009; Molenaar, 2004). Also central to the increasingly popularity of the case study is the growth of narrative and life-story methods for making sense of individual lives (McAdams, 2006). Within this broad approach, a growing number of researchers are employing case studies to illustrate and explore how individual human beings makes sense of their own lives in story, dialogue and narrative (e.g. Holstein \& Gubrium, 2012; McAdams, Josselson, \& Lieblich, 2006; Smith, 1999).

Research on the theory of emerging adulthood is one area where case studies have a notable foothold. Arnett (2004) employed case studies to exemplify aspects of the theory of emerging adulthood in relation to real lives, and other emerging adulthood phenomena have been explored via case studies, as we describe below (e.g. McAdams, 2013; Schachter, 2005). Van Dulmen (2013) has emphasized the importance of moving emerging adulthood research forward by innovating in data collection and study design. Case study work lends itself well to such innovations, for time-consuming data collection methods such as diaries, archival records, written narratives, third-party data from significant others, and observation can be used (and combined) with less strain on resources and time than in large-sample research.

The increasing receptivity to case studies in psychology is encouraging, but it is still a rare sight to see a case study mentioned within a mainstream psychology journal. We argue that 
this is because the theoretically-formative functions of the case study are not widely communicated and understood (Carlson, 1988). In order to clarify and expand the functional roles of case studies in emerging adulthood research, we outline four functions that they can serve; case-based theory development, individual-level theory testing, theory exemplification and idiographic psychobiography. For each one, we present illustrative examples from published research on emerging adulthood and also provide hypothetical ones. We explore the epistemological rationales, methodological processes and logistical challenges for each function. First, we explore a validity criterion that provides a generic rationale for case study work; that of specifiability.

\section{Specifiability - an external validity criterion for case study research}

The criterion that a research finding must be generalizable is central to scientific psychology, and rightly so. Science from the outset has looked to develop theories and laws that hold true across time and space for multiple phenomena, and psychology has adopted this objective, which can be traced all the way back to Platonism and the idea of singular or latent forms underling the varied phenomena and plurality of nature (Burtt, 2003). A generalizable theory or model in psychology is one that is held to represent some underlying commonality that human beings and groups share, which extends beyond the sample(s) upon which original research was based, to others in time and space. This enhances external validity, which in turn facilitates prediction and intervention in novel situations (Lewin, 1951).

But generalizability is not sufficient for external validity. Psychological theories and models, as well as showing generalizability, must also show specifiability. A theory/model that has good specifiability can be used to predict and understand and explain not just people on average, but also to predict one particular person. Specifiability matters because psychologists in the applied domain most often work with individual persons rather than with groups, so theories and models that have specifiability have clinical and applied utility. Furthermore, a specifiable theory has academic merit: A specifiable theory is likely to capture a formative (or even 
fundamental) process or structure that exists within the brain or mind, hence it shows up at the individual level and not merely at the aggregate level (Lamiell, 2003; Robinson, 2012).

A psychological theory may have good generalizability but poor specifiability. In statistical terms, this means that it will predict differences in means between groups, or predict group-level correlations, but not show accurate prediction or understanding at the individual level. In the standard quantitative method of psychology, this issue is common, for data are aggregated into groups for testing. Group-level differences (as shown by t-tests or ANOVA-based statistics), or group-level correlations, will struggle to be usefully or reliably predictive or explanatory at the individual level, unless the effect found is very strong. In moderate to low effect size findings, which are typical for psychology, many (or sometimes most) individuals within a group will not conform to its central tendency. For example, emerging adults have been found to report higher mean-levels of stress than adults in older age groups (Stone, Schwartz, Broderick, \& Deaton, 2010), and have been found to show a higher mean-level of personality change than older adults (Roberts, Walton \& Viechtbauer, 2006), but those differences are at the mean level, and a mean does not necessarily reflect the majority of a sample (a difference in means can be the product of a minority of cases within one group who score particularly high or low). In relation to the above findings there will therefore be a large proportion of young adults and older adults (maybe the majority) who do not conform to that average tendency with regards to differences in stress levels or personality change, even though the average tendency may likely be replicable and reliable across different samples at the group level. Thus the above findings may be generalizable but not specifiable.

How does one ensure that a theory has good specifiability? Case studies are the answer. A researcher can use case studies to develop, test, exemplify and apply theory in ways that provide clear and convincing evidence that the theory applies to individual lives in predictive, explanatory and interpretive ways. We now describe four ways of using case studies, all of which help enhance the specifiability of a theory or model. 


\section{Four functions of case studies in emerging adulthood research}

\section{Function 1: Case-based theory development}

Developing theory through case studies has a long heritage in psychology. Typically the researcher will use a series of individual cases in this work, each of which is presented separately. Classic examples of case study theory development include Pavlov's (1927) work on classical conditioning, and the work on problem solving by Newell and Simon (1972). These famous studies developed a new theory, and both of these theory development processes were highly intensive processes lasting years. This level of ambition is by no means necessary for the researcher who aspires to case-based theory development. A good and less resource-intensive option for many researchers who are interested in exploring the theory-development function of case studies is to try to continue developing an existing theory by extending it, qualifiying it or adding new depth and richness to it.

Any case-study led theory development work requires two steps: (a) a within-case (intraindividual) analysis of each contributing case, followed by (b) a cross-case search for common patterns (Eisenhardt, 1989). In qualitative research, these two steps can be illustrated by the work of Robinson and colleagues on the development of a theory of early adult crisis, which was in part based around case studies. The researchers initially analyzed the descriptions of crisis events given by 16 individuals between the ages of 25 and 39 (Robinson \& Smith, 2010a). Each crisis was analyzed individually first using within-case analysis, and this was followed by a cross-case analysis that extrapolated the following four common phases across the participants: (1) feeling locked in to a relationship, job or both, (2) a painful separation from the life structure that was centered around that commitment, (3) a trial-and-error exploratory period, and (4) a resolution into a new more intrinsically satisfying life structure. Following this method, an individual case was interviewed twice, then analyzed and reported as a single case (Robinson \& Smith, 2010b), in order to help develop the inner dynamics of the crisis model in relation to authenticity and 
persona. Following this, six individuals were interviewed to explore the cross-case validity of the themes that emerged from the case study (Robinson \& Smith, 2010c).

Theory development using case studies can be conducted using quantitative data too, using different processes of within-case analysis followed by cross-case analysis. There are specialized and complex statistical methods for this process (e.g. Molenaar, Sinclair, Rovine, Ram, \& Corneal, 2009), but given that within-case statistical analysis is still not widely used amongst academics and students, here we provide a few simple pointers for how a quantitative analysis package such as SPSS can be used to conduct within-case analysis. It differs in how the rows of the statistics spreadsheet are used. In standard group-based quantitative analysis, each row represents one individual in the sample. Nominal variables in the dataset allocate participants into groups or conditions.

In contrast, when conducting a within-case analysis, one spreadsheet represents repeatedmeasures data from just one person. Each row of the spreadsheet represents an occasion on which data has been gathered - row one is $\mathrm{T} 1$, row two is $\mathrm{T} 2$, etc. Continuous variables are shown in columns in the same way as in a group-based analysis, while nominal variables represent discrete phases, conditions or environments that the case study participant encountered over the time period studied, for example if the study was experimental, a variable could be used to allocate occasions into three phases conditions; pre-intervention, intervention and post-intervention.

A hypothetical example will help to illustrate this for an emerging adulthood study. Imagine a longitudinal case study in which a single participant provides data on their work status (employed / studying / unemployed), stress levels (scores on a single item with a Likert scale) and a weekly diary rating of recent level of identity exploration (1-7), on 50 occasions over a period of two years between the ages of 18 and 25 . The resulting spreadsheet will have 50 rows to represent the 50 data collection occasions and three columns to represent the three variables of work status, stress and identity exploration. Work status would be coded in a column using a nominal variable, while stress and identity exploration would be scored using continuous data. If 
a negative correlation is found between stress and identity exploration over the 50 measurement occasions, the resulting $r$ value represents intra-individual co-variation. It would indicate that there is a linear relationship between these two variables at the individual level over time. An analysis could also compare identity exploration levels across work status conditions, using an ANOVA. If a difference was found in identity exploration level across the three work status categories, this may suggest a systematic relationship between whether the person was working, studying or unemployed, and the amount of identity search they engage in.

In order to inductively establish the generalizability of any resulting within-case quantitative findings, the researcher would need to replicate the case study on a series of different cases, and establish the proportion or group of persons for whom a particular within-case pattern or effect holds, and make generalizations accordingly. In rare instances, cross-case analysis may show that all participants of a particular sample may show the same effect, in which case a strong assertion of an invariant commonality or general law may be warranted, which can be shown at the individual level as well as the group level, and thus shows good specifiability (Robinson, 2011).

\section{Function 2: Individual-level prediction testing}

Just as case studies can play a role in developing theory, they can be used to test an existing theory, by assessing the accuracy of predictions that stem from the theory. Two kinds of basic prediction can be made from psychological theory. The first is the group-probabilistic kind, which requires group-aggregate research to be tested, and the second kind is the individual-level prediction, which is best tested on case studies. Both kinds of prediction are important and useful, but psychology currently focuses on the former almost to the exclusion of the latter (c.f. Robinson, 2011). A theory has good specifiability when it can make reliable predictions about specific individuals, and we argue that specifiability is as important a validity criterion for theory as generalizability, because a useful and accurate psychological theory must tell us predictable information about individuals, in order to be of epistemic and practical use (Kluckhohn \& 
Murray, 1953). If both group-probabilistic and individual-level predictions are supported for the theory of emerging adulthood, this suggests that the theory has good generalizability and good specifiability. Here we describe examples of both kinds of prediction that stem from the theory of emerging adulthood, and the rationale for testing both.

Arnett's $(2000 ; 2004)$ theory of emerging adulthood contains a number of interlinked postulates about the dynamics of life and development in the 18-25 age range. Among these, the theory stipulates that emerging adulthood is the peak period of the lifespan for (1) identity exploration (in love, work and worldviews), (2) social instability (in relationships and residence), (3) a focus on self (in narrative and goals), (4) a feeling of being in-between (in social status), and (5) an optimistic sense of future possibility. The theory also states that emerging adulthood occurs to a greater degree in industrialized or post-industrial countries than in other countries. A number of group-probabilistic predictions can be made based on these postulates: Firstly one would predict that in the $18-25$ age range, the above five features would be more prevalent in wealthy industrialized countries compared with developing economies. Secondly, one would predict that a sample of emerging adults would show a greater concentration in extent and intensity of all these five features compared with samples of adolescents and of adults in later age ranges. These predicted group-level differences will manifest at the aggregate level and so are best tested with classic group-based quantitative methods. Because the predictions are phrased in a general way about 'emerging adults on the whole', it will be crucial that the selected sample of participants is representative of their age cohort. External validity of the study will be at stake if the sample is biased or limited in some way.

This kind of group-based hypothesis does not exhaust the predictions that can be made from the five core features of emerging adulthood. The theory is formulated to pertain to individual lives as well as groups, and has been illustrated using individual cases by Arnett (2004), so one can make predictions about individuals too. When forming a specific hypothesis 
about someone, rather than about everyone or people in general, success of the prediction pertains to that person only, and so generalization is not an issue (Mook, 1983).

An example prediction formulated in this way would be as follows: Joe is 18 and living in the UK. His parents are professional white British and are categorized in a relatively high socioeconomic group. We can predict the following, based on the identity aspect of the theory of emerging adulthood: Over the forthcoming seven years, Joe will show elevated levels of exploration in matters of social identity, relationships, personal goals, beliefs and values, and will likely defer major commitments, such as marriage and parenthood, to enable such exploration. This particular prediction is more challenging than the group-probabilistic kind - the theory must have a higher degree of precision to be tested in this way. There is no way to logically test such a prediction by recourse to a group - it has to be tested through longitudinal data collection from Joe. If the prediction were supported, the theory would be buttressed as one that can make specifiable predictions. If the prediction was not supported, and the selected individual showed no features of emerging adulthood, this would provide an interpretative challenge for the researcher: why have the predictions failed in this instance? Is it a matter of the person's religion, financial situation, culture or upbringing? What has led him to bypass the proposed normative identity explorations of emerging adulthood? One case is not enough to present a serious challenge to a theory, but if such individual-level predictions were tested repeatedly on a series of cases, and shown to be problematic on multiple occasions, the case studies together would cumulatively challenge the theory.

\section{Function 3: Theory exemplification}

Theories and models in psychology are verbal-mathematical abstractions that show the commonalities in human minds and behaviors. In order to maximize parsimony and usefulness, a theory should be simplified down to a set of verbal postulates, a box-and-arrow diagram, a formula or some other schematic. The resulting abstract theory can easily become divorced from the individuality, imperfection and idiosyncrasy that real life entails, and so psychology needs to 
constantly build clear bridges between the simplified abstractions of theory and the fleshed-out particulars of real life, to prevent it from suffering a 'reduction to the abstract' (Maslow, 1966, p.87).

Theory exemplification research aims to show how a theory or concept manifests within the individual case, in so doing creating important links between abstraction and the particular (McAdams \& West, 1997). Within this function, there is no testing of an explicit prediction; instead there is the more holistic aim of bringing the theory to life for others to comprehend better as an integrated totality in context. In his book Emerging Adulthood: The winding road from the late teens through the twenties, Arnett (2004) promotes this function of case studies to exemplify and illustrate the axioms of emerging adulthood:

"...there is much to be gained from describing individuals, so that we can see what a complete life looks like in emerging adulthood. If we only combined isolated parts from the interviews, we would never see how all of the parts fit together. By describing several people in detail, we can get a full sense of what it is like to be an emerging adult in all of its complexity...The profiles...will serve to illustrate the essential characteristics of emerging adulthood...by connecting them to real lives of emerging adults." (Arnett, 2004, p.28)

An example of theoretical illustration is Schachter's analysis of a single case study as a demonstration of how context and culture interplays in identity formation during emerging adulthood (Schachter, 2005). In this paper, a single case was used to demonstrate how cultural context molds identity development at multiple levels and also creates diverse notions of what a mature identity might look like. The individual in question was an Israeli Jewish Orthodox young man named Gil, who left school and first went to a yeshiva (a religious school) before going into the army and then to university to study science. He encountered a nexus of pre-modern, modern and postmodern contexts, all of which shaped his identity. He ended up finding an identity configuration that allowed for this juxtaposition, by accepting religion and science as 'bubbles' 
that do not have access to the whole truth, and by developing a postmodern worldview that holds truths are local and provisional, and that sees identity as provisional and dynamic too. This case was used by the author to illustrate a number of theoretical postulates, rather than to develop or test those theories. However the case is an implicit challenge to theory, insofar as the paper champions the importance of considering multiple levels of context in matters of emerging adult identity.

Another example of theoretical illustration in emerging adulthood research is the use of data from the biographies of Barack Obama and George W. Bush to convey how the developmental challenge of gaining life authorship manifests in the emerging adult period of these two presidents (McAdams, 2013). The two cases highlight the challenges specific to their individual circumstances, and also illustrate the common challenge of gaining life authorship in terms of exploring, and then regulating, a consistent identity through an interplay of culture and self. They both show how continuity and consistency of identity can be manifest in narrative form, in ways that lead to generativity in midlife.

When using and evaluating interventions to help emerging adults, case studies can be used to exemplify and illustrate theory in a more prescriptive sense, by illustrating and conveying best practice - in other words, by showing how to optimally apply theory to help individuals or groups. This demonstration of best practice has a strong heritage of case study work in therapeutic and clinical journals. For example, Bishop et al. (1986) provided an in-depth casespecific report on the use of cognitive therapy to help an emerging adult with psychotic depression. The paper's aim was not to develop or test theory, but rather to provide an in-depth exemplification of how clinical theory and process can best be used with a young adult client in practice.

\section{Function 4: Idiographic psychobiography}

The aim of psychobiography is to understand or explain an individual person, by gaining rich data from multiple sources, and analyzing it through the lens of existing theory. Data for a 
psychobiography may include archival records, diaries, letters, interviews with third parties, and interviews with the individual in question. The resulting report is typically structured as a psychological narrative that traces the development of important psychological constructs in one individual's life over time (e.g. McAdams, 2006; Schultz, 2005). Early psychobiographies were criticized for an overreliance on psychoanalytic speculation (e.g. Murray, 1938); however recent years have witnessed important theoretical and methodological advancements in the application of psychological theory to biography (e.g., Elms, 1994; Schultz, 2005). As illustrated in the Handbook of Psychobiography (Schultz, 2005), contemporary practitioners draw widely from evidence-based psychological theories to explore the lives of their subjects in depth.

Psychobiography is an exemplar of idiographic method. The terms nomothetic and idiographic were coined by the philosopher Windelband in the nineteenth century to distinguish science from the humanities (Lamiell, 1998; Robinson, 2012). Shortly after, they were imported into psychology to refer to different methodologies within the discipline (Munsterberg, 1899). In psychology, the label nomothetic was used to designate all methods that aim to develop or test general predictions, laws or theory, while the label idiographic was applied to research that aimed to understand an individual phenomenon using existing theory (Robinson, 2011).

Idiographic research has been criticized in the past for being anti-scientific (Nunally, 1978), on the basis that natural science is nomothetic and not interested in individuality per se. Such a criticism is based on a misconception of science as purely nomothetic. In fact, natural scientists not only seek general laws, but also go to some lengths to explore individuality in the natural world (Munsterberg, 1899). For example, cosmologists go to great lengths to explore the individual planets of our solar system. Each planet has been surveyed in great detail to develop a comprehensive map of the planet's topography, atmosphere and electromagnetic field. The findings from each planet will not be generalizable to other planets, but that is not a problem, for part of science's task is to catalogue the manifest world and the phenomena that comprise it, from galaxies and planets to trees and insects. Given that there are under ten planets in the solar 
system, each one can be studied in detail, but with human beings, an individual chosen for idiographic work will need to be purposively selected from billions of potential participants. The choice for psychobiography is typically based on the significance of the person to history or contemporary society. Individuals who have been selected for psychobiography include famous religious figures such as Luther (Erikson, 1958), Ghandi (Erikson, 1969) and Muhammad (Sina, 2008); political leaders such as George W. Bush (McAdams, 2011) or Saddam Hussein (Post, 2013), adventurers such as Dodge Morgan (Nasby \& Read, 1997) and serial killers such as Jeffrey Dahmer (Chéze, 2009).

Erikson's case study of Martin Luther is one of the more famous works of psychobiography to focus on the emerging adult life stage (Erikson, 1958). Entitled Young Man Luther, the work studied Luther's life between 1505 and 1517, which corresponds to Luther's 20s and 30s. During this 12-year period, Luther changed from being a retiring monk to an energetic church reformer, evidencing a personal crisis including sexual temptations, a conflict with his father, and resulting radical shifts in identity that are associated with emerging adulthood theory.

The central rationale and justification for a psychobiography is to develop an informed understanding of an individual. This is achieved by using psychological theory to interpret and make sense of the biographical data, a logical process termed abduction (Minnameier, 2010). Abduction involves the provision of an explanation or interpretation of data, events or facts by applying a theory, and showing the facts to be a manifestation of some aspect of the theory.

Abduction may involve considering competing theories and explanations for a particular occurrence. A seminal example of this with a case study is Runyan's (1981) study of Van Gogh which sought to explain why, at the age of 35 , he cut the lower half of his left ear off and took it to a brothel where he gave it to prostitute saying 'keep this object carefully'. The event occurred when Van Gogh was pursuing romantic attachments and a stable career. As such, the narrative resonates with features of emerging adulthood and the emotional struggles that lead some young adults to self-harm. Runyan posits thirteen possible explanations for the act, some of which have 
been postulated before by biographers. These include: Van Gogh's frustration over the engagement of his brother Theo against his own continued lack of a partner; an unconscious desire to maintain the attention of his brother, to whom he was very attached; a psychotic attack in which he was hearing voices and he believed that cutting the ear off would make the voices go away; the fact that in bullfights (which Van Gogh had witnessed many times) the successful matador is given the ear of the bull, which he then gives to the woman of his choice; or the fact that there is a scene in the bible where Simon Peter cuts off the ear of a servant, which Van Gogh had tried to paint once. Runyan set out a protocol for evaluating alternative interpretations by testing their validity against the evidence available. He suggests that competing explanations should be evaluated against the criteria of (a) logical soundness, (b) comprehensiveness in accounting for the various aspects of the event, (c) survival of tests that attempt to refute the explanation, (d) consistency with evidence available, and crucially (e) their consistency with general knowledge about human functioning, at biological, psychological and/or social levels. A theory that shows good abductive power in interpreting case studies will be able to explain a wide range of events in ways that adhere to these criteria.

Abductive interpretation of idiographic experiences and changes within the 18-30 age range provides an important function for theories of emerging adulthood and related phenomena. In George W. Bush and the Redemptive Dream, McAdams (2011) examined the development of Bush's personality at three different theoretical levels - basic dispositional traits, central motivational conflicts, and the internalized personal narrative that Bush ultimately constructed to make sense of his own life in time. The case study of the $43^{\text {rd }}$ President of the United States exemplified the dynamics of high lifelong extraversion and low openness to experience, making for a high-energy and impulsive leader who trusted his own gut instincts over reason and deliberation. Bush's idealization of his own father and his desire to defend him against all enemies ran from emerging adulthood onwards and proved to be an important factor behind his decision to launch a pre-emptive attack on Iraq in the spring of 2003. Layered over his traits and 
motivational goals, moreover, was a personal narrative of atonement and redemption that Bush developed in his late 30s, a story that he both used for his own psychological integration and projected onto world events. McAdams (2011) shows how the traits, goals, and story all worked together in Bush's personality development, in concert with a complex of external events and situational influences, to launch his political career and to inform many of the decisions he made as president, for better and for worse.

\section{The interdependence of the four functions}

The four functions of case studies have been presented throughout this article as separable and distinct, for the purposes of clear exposition, but they are in fact interdependent and interactive. A single study can employ more than one functional aim from the outset, and one function can evolve into another over the course of a research investigation. For example, individual-specific prediction testing can move towards a theory development objective if the problems highlighted by unsupported predictions can be solved by amending the theory. Robinson and colleagues tested the aforementioned model of early adult crisis with a further sample of individuals aged 25 to 35 (Robinson, Wright \& Smith, 2013). During the interviews, participants were shown a diagram of the model and asked which parts fitted their own crisis and which did not, and to discuss this. The result of this theory testing was a development of the theory to include a separation of Phase 2 into Phase 2a (Separation) and Phase 2b (Time Out), as well as including a 'fast forward loop' for those who miss out Phase 3 and a 'relapse loop' for those who temporarily resolve the crisis but then move back into another Phase 2 pattern.

As another example, psychobiography may start with an idiographic objective, but can lead to theoretical insights or theoretical exemplifications as analysis progresses, as illustrated above by the work by McAdams $(2011 ; 2013)$ on George W. Bush. The work was initially an extensive psychobiography of the man and his development, but was later used to exemplify the notion of narrative life authorship in emerging adulthood. In summary, there is much give-and- 
take between these four functions, and so they should not be viewed as discrete categories, but as four streams that feed into each other when working with case studies.

\section{Sampling in case study research}

When conducting a case study, the process for selecting a participant can be purposive or random, or a combination of these. There are a number of recognized purposive strategies for selecting cases (Robinson, 2014): If the objective is theoretical development, a strategy of intensity sampling can be used, which purposively selects a person who is assumed or known to be insightful, comprehensive, articulate and/or honest (Miles \& Huberman, 1994). This procedure was used by Schachter (2005) in the aforementioned case study of context and identity. The single case was chosen from a larger sample of narratives because of the richness and diversity with which the participant described his identity and development.

If the objective of a case study is individual-level prediction testing, a deviant case strategy may be used, in which an individual who is considered likely to problematize the theory is selected to explore the limits or problems inherent in a theory (Robinson, 2014). If the research objective is theory exemplification, a typical case strategy (also called emblematic case or paradigmatic case) could be used (Miles \& Huberman, 1994) - here the person is chosen precisely because he/she is a typical example of a theory, or an aspect of a theory. For example Arnett (2004) selected four individuals to exemplify what it is like to be an emerging adult in the USA as they were exemplars of exploration, instability, self-focus, feeling in-between and having a strong sense of possibility.

Finally, if the objective of the research is psychobiography, an individual will be selected non-randomly, usually for his or her historical, contemporary or theoretical significance - this purposive strategy is termed significant sampling (Simonton, 1999). The basis of what constitutes significance will be specific to a researcher's objectives, but in all instances it will be a rational inference that understanding the individual in depth may provide utility to research, history, policy or intervention. 
Purposive sampling strategies and random strategies are not mutually exclusive and can in some instances be used in combination. For example, for a case study by Robinson and Smith (2010a or b) on early adult crisis, intensity sampling was combined with random sampling. Firstly, a shortlist of individuals was selected from a previous interview study based on intensity sampling. The shortlist included all those who had provided a comprehensive account of both the inner and outer dimensions of their crisis experience. One person was then selected randomly from this information-rich subgroup (Robinson \& Smith, 2010b).

\section{Conclusions}

Greenwald (2012) argues that method and theory in psychology are deeply interdependent, and that methodological shifts often drive theoretical advances. We contend that case studies, if embraced more widely and systematically by emerging adulthood researchers, can have a variety of positive effects on theory. Bringing case-based theory development work into a central role will help to ensure that theories and models are neither just group-aggregate associations nor just taxonomic groupings, but are specifiable regularities that reflect intraindividual structures and processes. Individual-level theory testing will help further ascertain whether such theories and models are applicable and relevant when attempting to predict or understand real people. Theory exemplification will help to build clear and explicit links between the untidy world of real emerging adult lives, and the abstracted realm of theory, and ensure a dynamic interplay between these. Finally, a greater practice of idiographic psychobiography will facilitate a wider appreciation of the logical technique of abduction, in applying competing theories to explain individual phenomena. 


\section{References}

Arnett, J.J. (2000). Emerging adulthood: a theory of development from the late teens through the twenties. American Psychologist, 55, 469-480.

Arnett, J.J. (2004). Emerging Adulthood: The winding road from the late teens through the twenties. New York: Oxford University Press.

Barlow, D.H., \& Nock, M.K. (2009). Why can't we be more idiographic in our research? Perspectives on Psychological Science, 4, 19-21.

Bishop, S., Miller, I.W., Norman, W., Buda, M., \& Foulke, M. (1986). Cognitive therapy of psychotic depression: A case report. Psychotherapy, 23, 167-173.

Burtt, E.A. (2003). The metaphysical foundations of modern science. London: Dover Publications.

Carlson, R. (1988). Exemplary lives: The uses of psychobiography for theory development. Journal of Personality, 56, 105-138.

Chéze, E. (2009). Jeffrey Lionel Dahmer: A psychobiographical study. Unpublished doctoral thesis, Nelson Mandela Metropolitan University.

Eisenhardt, K.M. (1989). Building theories from case study research. Academy of Management Review, 14, 532-550.

Elms, A.C. (1994). Uncovering lives: The uneasy alliance of biography and psychology. New York: Oxford University Press.

Erikson, E. (1969). Ghandi's Truth. New York: W. W. Norton.

Erikson, E. (1958). Young man Luther: A study in psychoanalysis and history. New York: W. W. Norton.

Greenwald, A.G. (2012). There is nothing so theoretical as a good method. Perspectives on Psychological Science, 7, 99-108. doi: 10.1177/1745691611434210

Holstein, J.A., \& Gubrium, J.F. (Eds.). (2012). Varieties of narrative analysis. London: Sage. Josselson, R., \& Lieblich, A. (Eds.). (1993). The narrative study of lives. Thousand Oaks, 
CA: Sage.

Kluckhohn C.E. \& Murray, H.A. (1953). Personality in nature, culture and society. Oxford, England: Knopf.

Lamiell, J.T. (1998) 'Nomothetic' and 'Idiographic' - Contrasting Windelband's understanding with contemporary usage. Theory and Psychology, 8, 23-28. doi: $10.1177 / 0959354398081002$

Lamiell, J.T. (2003). Beyond individual and group differences: Human individuality, scientific psychology, and William Stern's critical personalism. London: Sage.

Maslow, A.H. (1966). The psychology of science. London: Harper and Row.

McAdams, D. (2006). The redemptive self: Stories Americans live by. Oxford University Press Inc.

McAdams, D.P. (2011). George W. Bush and the redemptive dream: A psychological profile. New York: Oxford University Press.

McAdams, D.P. (2013). Life authorship: A psychological challenge for emerging adulthood, as illustrated in two notable case. Emerging Adulthood, 1, 151-158.

McAdams, D.P., Josselson, R., \& Lieblich, A. (Eds.). (2006). Identity and story: Creating the self through narrative. Washington, DC: APA Books.

McAdams, D., \& West, S. (1997). Introduction: Personality psychology and the case study. Journal of Personality, 65, 757-783.

Miles, M.B., \& Huberman, A.M. (1994). Qualitative data analysis: An expanded sourcebook. London: Sage.

Molenaar, P.C.M. (2004). A manifesto on psychology as idiographic science: bringing the person back into scientific psychology, this time forever. Measurement: Interdisciplinary Research \& Perspective, 2, 201-218.

Molenaar, P.C.M., Sinclair, K.O., Rovine, M.J., Ram, M. \& Corneal, S.E. (2009). Analyzing 
developmental processes on an individual level using nonstationary time series modelling. Developmental Psychology, 45, 260-271.

Mook, D.G. (1983). In defense of external invalidity. American Psychologist, 38, 379-387.

Minnameier, G. (2010).The logicality of abduction, deduction and induction. In: M.Bergman, S. Paavola, A.V. Pietarinen, \& H. Rydenfelt, (Eds.) Ideas in action: Proceedings of the applying Peirce conference (pp. 239-251). Helsinki: Pragmatism Network.

Munsterberg, H. (1899). Psychology and history. Psychological Review, 6, 1-31.

Murray, H.A. (1938). Explorations in personality. New York: Oxford University Press.

Nasby, W., \& Read, N. (1997). The life voyage of a solo circumnavigator: Integrating theoretical and methodological perspectives: Introduction. Journal of Personality, 65, 787-794.

Newell, A., \& Simon, H.A. (1972). Human problem solving. Englewood Cliffs: Prentice Hall.

Nunnally, J.C. (1978). Psychometric theory (2nd ed.). New York: McGraw-Hill.

Pavlov, I.P. (1927). Conditioned reflexes: an investigation of the physiological activity of the cerebral cortex. London: Oxford University Press.

Post, J.M. (2013). Psychobiography: The child is father of the man. In L. Huddy, D.O. Sears, \& J.S. Levy. The Oxford Handbook of Political Psychology (2 ed.). pp. 459-488. NY, US: Oxford University Press. DOI:10.1093/oxfordhb/9780199760107.013.0015

Roberts, B.W, Walton, K.E., \& Viechtbauer, W. (2006). Patterns of mean-level change in personality traits across the life course: A meta-analysis of longitudinal studies. Psychological Bulletin, 132, 1-25.

Robinson, O.C. (2011). The idiographic / nomothetic dichotomy: tracing historical origins of contemporary confusions. History \& Philosophy of Psychology, 13, 32-39.

Robinson, O. (2012). A war of words: the history of the idiographic/nomothetic debate. The Psychologist, 25, 164-167.

Robinson, O.C. (2014). Sampling in interview-based qualitative research: A theoretical and 
practical guide. Qualitative Research in Psychology, 11, 25-41. DOI:

$10.1080 / 14780887.2013 .801543$

Robinson, O.C. \& Smith, J.A. (2010a). Investigating the form and dynamics of crisis episodes in early adulthood: The application of a composite qualitative method. Qualitative Research in Psychology, 7, 170-191.

Robinson, O.C \& Smith, J.A. (2010b). A case study of pre-midlife crisis: An extraordinary transformational episode in an ordinary life. In D. Robinson et al. (Eds.) Narrative, memory and ordinary lives (pp. 145-157). Huddersfield: University of Huddersfield Press.

Robinson, O.C. \& Smith, J.A. (2010c). The stormy search for self in early adulthood: Developmental crisis and the dissolution of dysfunctional personae. The Humanistic Psychologist, 38, 120-145. DOI: 10.1080/08873267.2010.485916

Robinson, O.C., Wright, G.R.T. \& Smith, J.A. (2013). The holistic phase model of early adult crisis. Journal of Adult Development, 20, 27-37.

Runyan, W.M. (1981). Why did Van Gogh cut off his ear? The problem of alternative explanations in psychobiography. Journal of Personality and Social Psychology, 40, 1070-1077.

Schachter, E.P. (2005). Context and identity formation: A theoretical analysis and a case study, Journal of Adolescent Research, 20, 375-395.

Schultz, W.T. (Ed.). (2005). Handbook of psychobiography. New York: Oxford University Press.

Simonton, D.K. (1999). Significant samples: The psychological study of eminent individuals. Psychological Methods, 4, 425-451.

Sina, A. (2008). Understanding Muhammad: A psychobiography of Allah's prophet. Felibri.

Smith, J.A. (1999). Identity development during the transition to motherhood: An interpretative phenomenological analysis. Journal of Reproductive and Infant Psychology, 17, 281-299. 
Stone, A.A., Schwartz, J.E., Broderick, J.E., \& Deaton, A. (2010). A snapshot of the age distribution of psychological well-being in the United States. Proceedings of the National Academy of Sciences of the United States of America, 107, 9985-9990.

Van Dulmen, M.H.M. (2013, October). Are we there yet? Moving the science of emerging adulthood into the next gear. TEDtalk presented at the $6^{\text {th }}$ Conference on emerging adulthood, Chicago (IL). 